Rev. Adm. Saúde (On-line), São Paulo, v. 20, n. 79: e207, abr. - jun. 2020, Epub 02 jun. 2020 http://dx.doi.org/10.23973/ras.79.207

RELATO DE CASO

\title{
Design Thinking aplicado à comunicação institucional de um hospital público de ensino do estado de São Paulo
}

Design Thinking applied to the institutional communication of a teaching public hospital of state of São Paulo

\section{Caroline Maiolini Mizael1, Ligia Mayumi $\mathrm{Abe}^{2}$, Ivisen Teixeira Lourenço ${ }^{3}$, Marisa Riscalla Madi ${ }^{4}$, Rosemeire Keiko Hangai ${ }^{5}$}

1. Enfermeira. Aprimoranda do Programa de Estudos Avançados em Administração Hospitalar e Sistemas de Saúde (PROAHSA) do Hospital das Clínicas da Faculdade de Medicina da Universidade de São Paulo (HCFMUSP). São Paulo SP

2. Biomédica. Aprimoranda do PROAHSA do HCFMUSP. São Paulo, SP

3. Administrador. Gestor de inovação e marketing do Centro de Inovação e Tecnologia do HCFMUSP. São Paulo, SP

4. Médica. Diretora do PROAHSA do HCFMUSP. São Paulo SP

5. Enfermeira. Coordenadora de ensino do PROAHSA do HCFMUSP. São Paulo SP

\section{RESUMO}

Introdução: O papel da comunicação na gestão organizacional é crescente e de extrema importância nos processos de mudança e na busca de melhores resultados, pois facilitam o processo de difusão de informações, que são estratégias para a conquista de objetivos comuns, por meio do conhecimento compartilhado. Com a intenção de investigar a falta de adesão dos profissionais aos meios de comunicação institucional e de propor melhorias neste processo, a fim de contribuir para o alinhamento de diferentes equipes aos desafios estratégicos e projetos da instituição, a pesquisa foi proposta. Objetivos: Analisar e propor melhorias na comunicação institucional através da 
abordagem Design Thinking. Métodos: Como uma abordagem, o Design Thinking possui a capacidade de combinar empatia no contexto do problema, de forma a colocar as pessoas no centro do desenvolvimento do projeto, criatividade para geração de soluções e razão para analisar e adaptar as soluções para o contexto. A fim de identificar as dores e os ganhos dos colaboradores em relação a comunicação institucional e a disseminação do planejamento estratégico do hospital e propor melhorias, foram desenvolvidas as seguintes etapas: imersão (acompanhamento e entrevista com colaboradores), ideação (brainstorming com profissionais de diferentes áreas do hospital e definição da estratégia de melhoria) e prototipação (validação da estratégia com os colaboradores). Resultados: Em relação a comunicação institucional, foram identificadas as seguintes dores: não há tempo na rotina de trabalho para atualizações; muitos e-mails; e-mails não relacionados com a sua área/setor; informações desinteressantes e não atrativas; comunicação verbal ineficaz; poucas reuniões voltadas para assuntos institucionais. E os seguintes ganhos: gostariam de participar mais das discussões e estratégias; informações individualizadas e personalizadas; maior interação pessoal; se sentir mais valorizado; ter rastreabilidade das informações; informações rápidas, simples e objetivas. A proposta de melhoria é a inserção de painéis interativos nos setores do hospital com as principais entregas do planejamento estratégico e suas metas. Trata-se de um produto que utiliza a gameficação (estratégia envolvendo jogos) para auxiliar e melhorar o processo de comunicação. Conclusão: O projeto proporcionou conhecer a experiência dos colaboradores da instituição com a comunicação interna e o quanto a sua falha prejudica no entendimento dos principais objetivos estratégicos da organização e consequentemente na dificuldade em alcançar metas, já que as equipes não entendem o planejamento estratégico e seus produtos. A abordagem Design Thinking foi uma ferramenta bastante apropriada para o projeto, pois possibilitou o desenvolvimento de empatia para com esses colaboradores e, dessa forma, desenvolver uma proposta de melhoria que atinja suas principais dores e ganhos sobre o processo de comunicação e disseminação do planejamento estratégico.

Palavras-chave: Administração Hospitalar; Comunicação; Inovação; Planejamento Estratégico.

\section{ABSTRACT}

Introduction: The role of communication in an organizational management has become extremely important in the process of change and to achieve better results. The dissemination of information became easier by sharing the knowledge, which is strategic to achieve common goals. In order to investigate the lack of adherence of professionals to the institutional communication media and to propose improvements in this process, to contribute the alignment of different teams to the strategic challenges and projects of the institution, the research was proposed. Objective: Analyze and propose improvements in the institutional communication using the Design Thinking approach. Methods: As an approach, Design Thinking has the ability to combine empathy with the context of the problem, putting the people at the center of the project 
development. The approach has creativity to create solutions and reason to analyze and adapt solutions to the context. To identify employees pains and gains about institutional communication and the dissemination of the hospital's strategic planning and to propose improvements, the following steps were developed: immersion (monitoring and interview with employees), ideation (brainstorming with professionals from different areas of the hospital and defining the improvement strategy) and prototyping (strategy validation with employees). Results: About the institutional communication, the following pains were identified: no time in work routine for upgrades; many e-mails; emails that aren't from your area/sector; uninteresting and unattractive information; ineffective verbal communication; few meetings about institutional subjects. And the following gains: would like to participate more in discussions and strategies; individualized and personalized information; more personal interaction; feel more valued; have traceability of information; fast, simple and objective information. The improvement proposal is the insertion of interactive panels in the hospital sectors with the main deliverables of strategic planning and its goals. It's a product that uses gamefication to help and improve the communication process. Conclusion: The project provided knowledge about the employees experience with the internal communication and how this failure impairs the understanding about main organization strategic objectives and consequently the difficulty to achieve goals, because teams don't understand the strategic planning and their products. The Design Thinking approach was a very appropriate tool for this project, because it enabled to develop the empathy with these employees and, from this, to develop an improvement proposal for the main pains and gains of the communication process and to disseminate a strategic planning.

Keywords: Hospital Administration; Communication; Innovation; Strategic Planning.

\section{INTRODUÇÃO}

O papel da comunicação na gestão organizacional é crescente e de extrema importância nos processos de mudança e na busca de melhores resultados. A comunicação organizacional facilita o processo de difusão de informações e é uma estratégia para a conquista de objetivos comuns, por meio do conhecimento compartilhado ${ }^{(1)}$.

A partir da comunicação se desenvolvem os demais processos organizacionais. O relacionamento entre os trabalhadores e a organização permite o alinhamento de estratégias para atingir os objetivos institucionais ${ }^{(2)}$. Portanto, a comunicação é fator de viabilização dos processos, demandando a coleta e divulgação constantes de informação ${ }^{(3)}$. 
No ambiente hospitalar, a comunicação de excelência contribui para o alinhamento da equipe médica, multiprofissional e administrativa para o desenvolvimento das ações propostas no planejamento estratégico do serviço, a fim de contribuir no alcance das metas estabelecidas para a qualidade assistencial, excelência do serviço e dos profissionais ${ }^{(4)}$. Além disso, a comunicação pode estabelecer um sentimento de pertencimento, compromisso com os resultados, metas e protocolos, espírito de equipe, entre outros fatores essenciais para a motivação dos colaboradores ${ }^{(5)}$.

Nos serviços de saúde, a comunicação assume pontos desafiadores. Os hospitais são instituições extremamente heterogêneas em relação às rotinas, aos profissionais, aos pacientes, procedimentos e processos administrativos. Desempenhar a comunicação em um ambiente tão plural, construindo identificação entre os profissionais e a organização, é o desafio que as instituições enfrentam atualmente. O principal desafio é atrair a atenção das diversas equipes de forma contínua, pois o volume de informações é cada vez maior, vindos de diversos setores da organização ${ }^{(5)}$.

O processo de inovação engloba etapas que orientam atividades desde a geração de ideias até a implementação da solução de problemas. O Design Thinking é uma ferramenta que vem sendo utilizada como um meio de desenvolver inovações nas organizações. Consiste em uma abordagem colaborativa de resolução de problemas, centrada no usuário, que gera inovação através de iteração e práticas criativas ${ }^{(6,7)}$.

O Design Thinking alcança soluções inovadoras por meio da compreensão do contexto. É uma ferramenta que visa a compreensão, visualização e descrição de problemas complexos para, através da criatividade, auxiliar no desenvolvimento de soluções para tais problemas ${ }^{(8)}$.

Com a intenção de investigar a falta de adesão dos profissionais aos meios de comunicação institucional do hospital do presente estudo e de propor melhorias neste processo com o uso de uma ferramenta da inovação, o Design Thinking, a fim de contribuir para o alinhamento das equipes aos desafios estratégicos e projetos da instituição, a pesquisa foi proposta.

\section{OBJETIVO}

O objetivo deste artigo é analisar e propor melhorias na comunicação institucional de um hospital público de ensino através da abordagem Design Thinking.

\section{MÉTODOS}

A abordagem metodológica utilizada foi o Design Thinking, que nos auxilia na imersão e no entendimento de parâmetros e padrões essenciais para criar soluções de melhor qualidade através de quatro etapas: imersão (entendimento), ideação (criação), prototipação (teste) e desenvolvimento (aplicação). 
Como uma abordagem, o Design Thinking possui uma capacidade de combinar empatia no contexto de um problema, de forma a colocar as pessoas no centro do desenvolvimento do projeto; criatividade para geração de soluções; e razão para analisar e adaptar as soluções para o contexto.

A fim de identificar as dores e os ganhos dos colaboradores em relação a comunicação institucional e a disseminação do planejamento estratégico do hospital, além de propor melhorias, foram desenvolvidas as seguintes etapas do Design Thinking: imersão (acompanhamento e entrevista com colaboradores), ideação (reunião de brainstorming com profissionais de diferentes áreas do hospital e definição da estratégia de melhoria) e prototipação (validação da estratégia com os colaboradores).

A etapa de desenvolvimento não foi realizada devido ao prazo estabelecido para a realização da pesquisa. A intenção foi identificar as dores e ganhos dos colaboradores em relação a comunicação institucional e propor melhorias. A seguir, serão descritas cada etapa desenvolvida.

\section{Imersão}

Nesta etapa foi desenvolvida a empatia com os funcionários, para entender a experiência e o envolvimento do colaborador com a comunicação institucional e seu entendimento do planejamento estratégico do hospital. Inicialmente, foi acompanhado um período de trabalho de um médico, um enfermeiro, um técnico de enfermagem e um técnico de radiologia.

Além de acompanhar um período de trabalho, foram feitas perguntas abertas e espontâneas para os colaboradores sobre sua rotina e comunicação. A observação e as conversas proporcionaram o conhecimento do público. A síntese das informações foi feita com o auxílio do Canvas Mapa de Empatia (Figura 1). A partir da síntese, foi criada uma persona para representar os funcionários e para a análise de suas dores e ganhos no processo de comunicação e disseminação do planejamento estratégico do hospital, com o auxílio do Persona Canvas (Figura 2). O Value Proposition Canvas (Figura 3) auxiliou na definição de dores e ganhos dos colaboradores

Figura 1. Canvas Mapa de Empatia. 


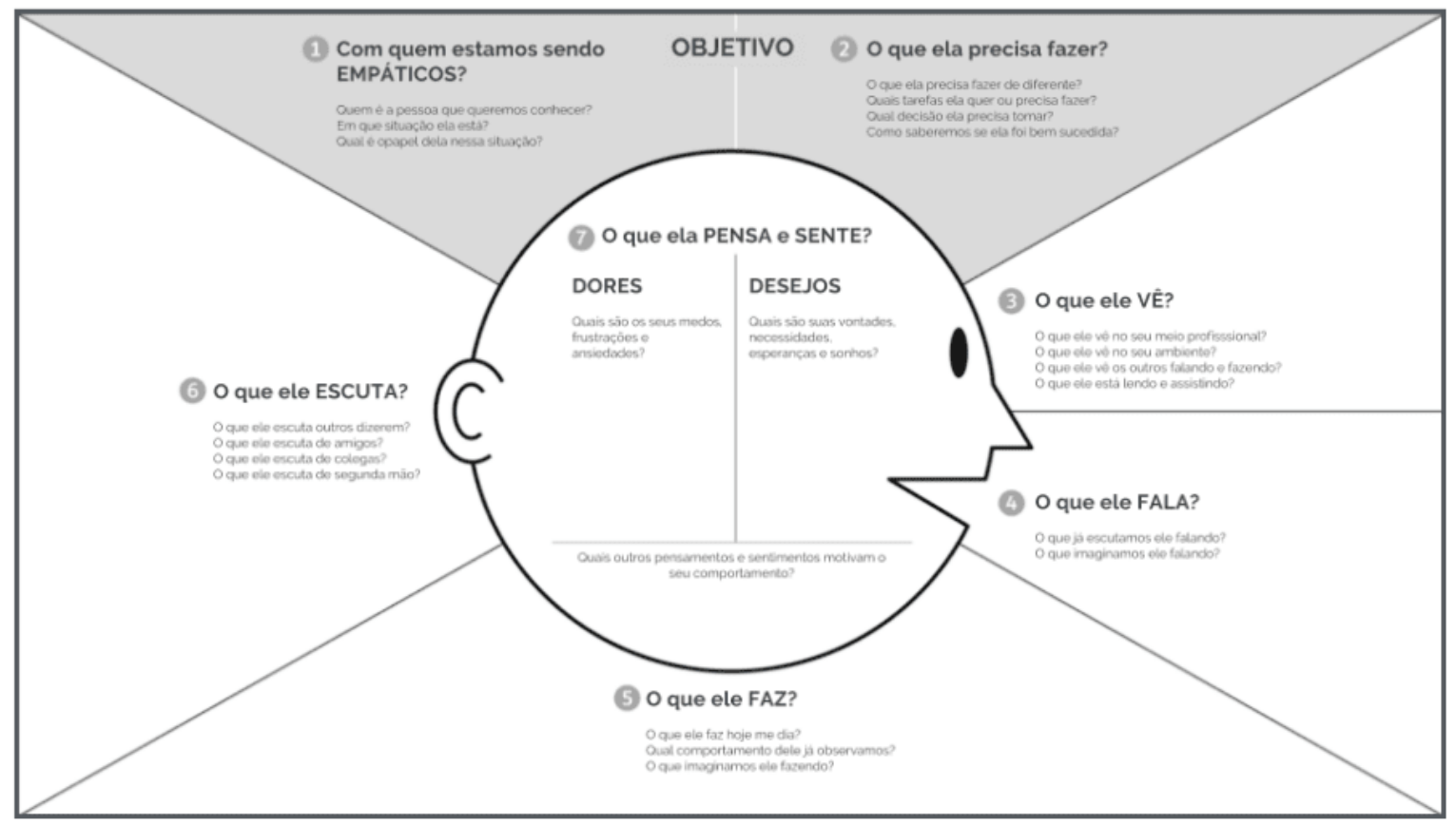

Fonte: analistamodelosdenegocios.com.br

Figura 2. Persona Canvas.

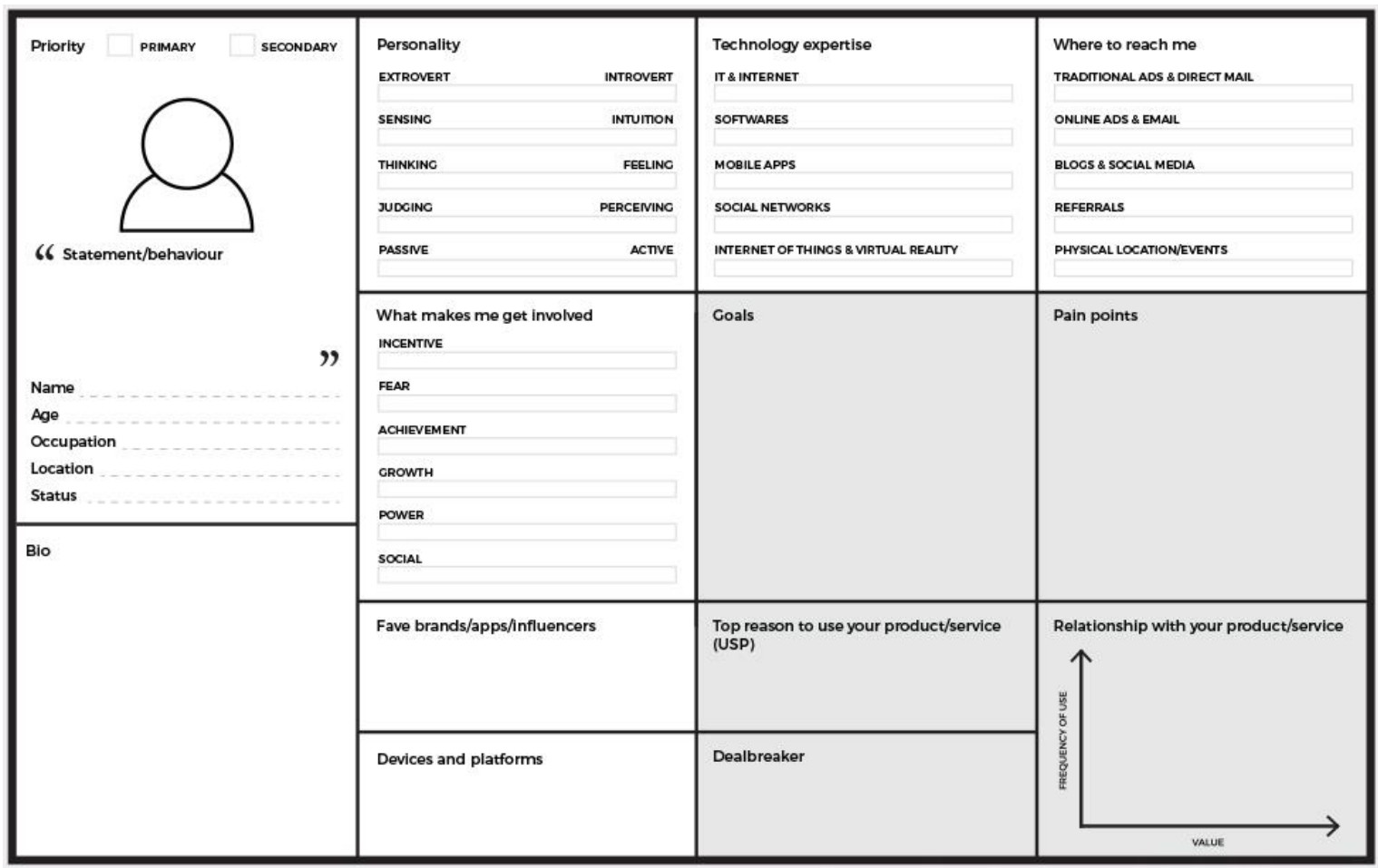

Fonte: businessdesigntools.com

Figura 3. Value Proposition Canvas 


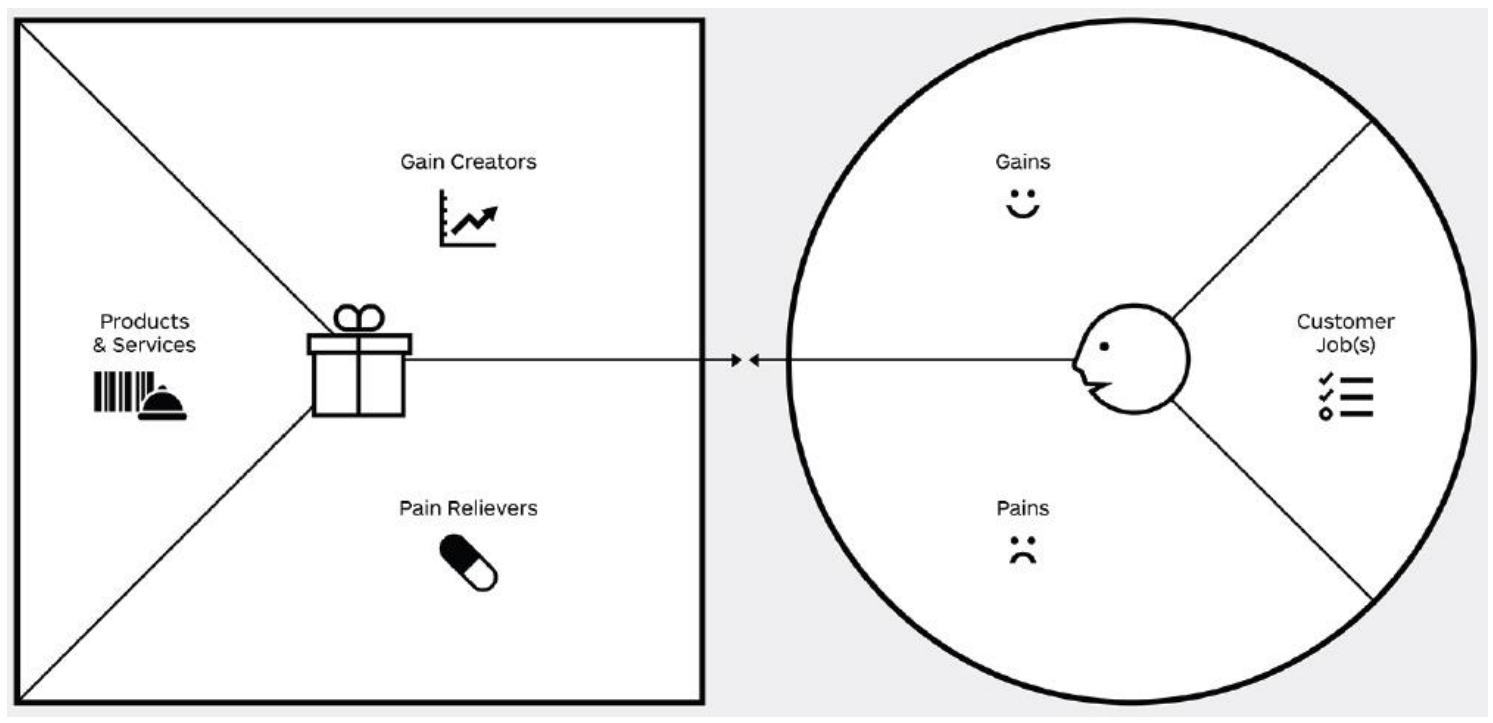

Fonte: strategyzer.com

\section{Ideação}

Nesta etapa, foi realizado um workshop de criação, onde estavam presentes profissionais de diferentes áreas para que cada um pudesse contribuir com sua expertise. As áreas representadas no workshop foram: Centro de Comunicação Institucional, Centro de Planejamento e Gestão, Centro de Engenharia e Arquitetura Hospitalar, Gestão de Pessoas, Centro de Inovação e Tecnologia, Centro de Comunicação de outro hospital público de ensino e os autores do projeto.

No workshop, foi proposto uma seção de brainstorming. Inicialmente, cada participante desenvolveu e escreveu o máximo de ideias que tiveram em 10 minutos e em seguida as ideias de cada um foram expostas e complementadas pelos outros participantes. Ao final, foram eleitas as 5 melhores ideias.

A etapa de criação foi norteada pela seguinte pergunta: "Como melhorar a disseminação de informações estratégicas na organização?"

Para o desenvolvimento do produto de melhoria, foram utilizadas quatro perguntas: Como funciona? Quem irá usar isso e quando? Por que melhora a experiência do funcionário? Como pode ser implementado?

\section{Prototipação}

Na prototipação foi realizada a validação da ideia desenvolvida na etapa anterior. A proposta foi apresentada para um grupo de colaboradores de um setor do hospital.

\section{RESULTADOS E DISCUSSÃO}


A seguir, serão descritos os resultados de cada etapa do Design Thinking desenvolvida.

\section{Imersão, etapa do entendimento}

Durante a primeira etapa, foram desenvolvidas a síntese das observações do cotidiano e a persona que representa o colaborador. A criação de personas é uma estratégia utilizada em marketing com o objetivo de aproximar o público da marca da empresa ${ }^{(9)}$. No caso em análise, a criação da persona foi utilizada como uma forma de desenvolver o perfil do colaborador para entender como eles se comportam, quais as demandas mais latentes (dores) e o que de fato faz diferença na experiência dos colaboradores do hospital (ganhos).

A criação da persona contribui para o processo de criação de conteúdos que ofereçam maior possibilidade de engajamento do público. Desta forma, foi criada uma persona: Nancy, que representa os profissionais assistenciais do hospital.

A persona, em conjunto com as observações do cotidiano deste colaborador, foram fundamentais para a identificação das iniciativas para a melhora da experiência dos colaboradores. A persona criada com auxílio do Persona Canvas e suas características, dores e ganhos estão visíveis na Quadro 1, também é possível visualizar as questões do Canvas Mapa de Empatia.

Quadro 1. Persona e questões do Canvas Mapa de Empatia.

\section{Persona}

Nancy, técnica de enfermagem, casada, 45 anos, 2 filhos, trabalha no hospital há 15 anos e tem outro emprego. Introvertida, passiva, intuitiva, racional e julgadora. Usa celular frequentemente, principalmente redes sociais. Participa de eventos e gosta de comunicação interpessoal.

\section{Mapa de Empatia}

\begin{tabular}{|l|l|l|l|}
\hline O que vê? & O que fala? & O que faz? & O que escuta? \\
\hline
\end{tabular}

Os funcionários observados utilizam o computador em sua rotina de trabalho, porém possuem pouco tempo para se atualizar em relação ao hospital através de leitura de e-mails, intranet, murais e painéis durante a rotina de trabalho. Não se interessam pela maioria das informações, pois grande parte delas não estão relacionadas à sua categoria profissional e setor de trabalho. Recebem uma quantidade massiva de e-mails e as informações em murais/painéis não são atrativas ou são informações administrativas do próprio setor, ou seja, não abordam o planejamento estratégico.

Os funcionários preferem que sejam realizadas reuniões com o gestor para discussão de informações administrativas, estratégicas e gerenciais, devido à possibilidade de esclarecer dúvidas, discutir diferentes opiniões e 
alinhamento da equipe como um todo, para evitar erros de compreensão e divulgação de informações erradas e/ou incompletas, porém referem a impossibilidade de reuniões periódicas devido a rotina atarefada na assistência.

Muitos dos colaboradores desconhecem o planejamento estratégico, metas e desafios traçados pelo hospital, porém já ouviu rapidamente através de meios de comunicação informais ("rádio peão").

No cotidiano, são pessoas que utilizam celular e computador como maiores meios de comunicação, que consomem informações navegando em sites de pesquisa e que utilizam redes sociais, que para o trabalho, consideram ser uma ferramenta eficiente para informações rápidas e objetivas, porém a informação não fica documentada, como no e-mail, por exemplo.

\begin{tabular}{|l|l|}
\hline Dores & Ganhos \\
\hline $\begin{array}{l}\text { Não há tempo na rotina de trabalho } \\
\text { para atualizações; }\end{array}$ & $\begin{array}{l}\text { Gostariam de participar mais das } \\
\text { discussões e estratégias (fazer } \\
\text { E-mails que não são de sua }\end{array}$ \\
área/setor; & Informações individualizadas e \\
Informações desinteressantes e não & personalizadas; \\
atrativas; & Maior interação pessoal; \\
Comunicação verbal ineficaz; & Sentir-se mais valorizado; \\
Poucas reuniões voltadas para & Ter rastreabilidade das informações; \\
assuntos institucionais. & Informações rápidas, simples e \\
& objetivas.
\end{tabular}

\section{Ideação, etapa da criação}

De posse da persona construída na etapa anterior, a próxima etapa foi o brainstorming, que é uma maneira de gerar ideias e novas alternativas (10). Nessa etapa, profissionais de diferentes áreas foram orientados a pensar em alternativas sem se preocuparem com possíveis restrições internas da empresa. Ou seja, a ordem dessa etapa era levantar o maior número de ideias sem se preocupar se elas eram passíveis de execução ou não. A Quadro 2 indica quais foram as ideias geradas para a solução do problema na comunicação institucional e as melhores ideias, que foram votadas pelos próprios participantes do brainstorming.

Quadro 2. Ideias de melhoria para comunicação institucional criadas no brainstorming. 


\section{Ideias do brainstorming}

- Time de colaboradores: "Peão falador";

- Gamificação sobre o planejamento estratégico;

- Vídeos rápidos com histórias sobre o planejamento estratégico (Storytelling);

- Canal em alguma plataforma de vídeos para hospedar os vídeos do storytelling;

- Pauta obrigatória do planejamento estratégico nos eventos/reuniões do hospital;

- Caricatura de cada colaborador comentando os objetivos estratégicos (balões de fala), em locais de convivência;

- Reuniões proativas;

- Painel nos setores com metas e entregas do planejamento estratégico;

- Jornal eletrônico;

- Material físico com os objetivos (crachá, prisma, lista);

- Banco de ideias pré, durante e pós planejamento com feedback;

- Aplicativo do planejamento estratégico;

- Objectives and Key Results (OKR's).

\section{Melhores ideias}

- Aplicativo do planejamento estratégico;

- Vídeos rápidos com histórias sobre o planejamento estratégico (Storytelling);

- Adesivos de emoji de cada colaborador comentando os objetivos estratégicos (balões de fala), em locais de convivência;

- Time de colaboradores;

- Painel a vista com metas e entregas do planejamento estratégico.

Após a sessão de brainstorming, foi desenvolvida a proposta de produto com o objetivo de aliviar as dores e desenvolver os ganhos em relação a comunicação e disseminação do planejamento estratégico do hospital.

A proposta desenvolvida originou-se da ideia de painel a vista com o planejamento estratégico e suas metas. Trata-se de um produto que utiliza a gamificação (estratégia envolvendo jogos) para auxiliar e melhorar o processo de comunicação para a disseminação as propostas do planejamento estratégico. Tendo como base quatro perguntas, a proposta foi estruturada de acordo com a descrição no Quadro 3.

Quadro 3. Proposta de produto para melhoria da Comunicação institucional. 


\section{Como funciona?}

- Cada setor terá um painel em formato de tabuleiro;

- Em cada setor, as categorias profissionais (médico, equipe de enfermagem, biomédicos e físicos-técnicos) criarão uma caricatura (animação) para representá-los no painel;

- O tabuleiro deverá conter as principais entregas do planejamento estratégico do ano;

- Cada categoria profissional, juntamente com a sua liderança, deverá traçar metas para contribuir com as entregas do planejamento estratégico;

- Conforme as metas forem sendo cumpridas, a animação percorre o caminho no tabuleiro, chegando mais próximo das entregas;

- Cada meta alcançada deve ser comemorada, muito importante a presença da liderança no setor para o feedback;

- Cada categoria (médico, equipe de enfermagem, biomédicos e físicostécnicos) terão as suas metas;

- O setor só completará o tabuleiro quando todas as categorias alcançarem as suas metas;

- Deve ter um painel geral para que os setores vejam a evolução entre si;

- As regras do jogo serão disseminadas por vídeos animados com as animações;

- Vídeos relacionando histórias de pacientes e o planejamento estratégico para chamar a atenção dos colaboradores.

\section{Quem irá usar isso e quando?}

Todas as categorias profissionais do hospital atuantes na assistência direta ao paciente: médico, equipe de enfermagem, biomédicos e físicos-técnicos estariam envolvidos nesse processo e os horários para reuniões e manuseio do tabuleiro seria determinado pelo setor.

\section{Por que melhora a experiência do funcionário?}

Essa proposta seria uma estratégia de aliviar as dores e estimular os ganhos identificados nesses funcionários em relação a comunicação e disseminação do planejamento estratégico, pois disponibiliza a participação dos funcionários nas discussões de metas e estratégias do instituto, a comunicação verbal com a liderança e a equipe estará presente, sem riscos de perda de informações, é uma forma personalizada, rápida e objetiva de se atualizar, não será necessário o uso do e-mail coorporativo e o painel estará sempre exposto para a consulta dos funcionários.

\section{Como pode ser implementado?}

Será necessário:

- Designer para o tabuleiro e animações;

- Empresa de mídia para vídeos;

- Definir regras do jogo; 
- Alinhar com as lideranças as entregas do planejamento estratégico no ano e, posteriormente, cada um com a sua equipe.

\section{Prototipação, etapa do teste}

Na prototipação, foi realizada a validação da ideia desenvolvida na etapa anterior. A proposta foi apresentada para um grupo de colaboradores de um setor do hospital. A reação à ideia do produto foi positiva e surgiram algumas contribuições. Foi sugerido que os vídeos relacionando histórias de pacientes e o planejamento estratégico fossem divulgados em um período suficiente para que os colaboradores possam entender o que é o planejamento estratégico e o impacto que seus produtos trazem para a experiência do paciente e assim, posteriormente, implantar o painel-tabuleiro.

Se o painel-tabuleiro fosse implantado sem uma prévia comoção do colaborador ao ver o impacto que o planejamento estratégico pode ter para os pacientes, talvez o jogo não seria desenvolvido com entusiasmo pela equipe. Portanto seria importante os vídeos terem um teor emocional e seja provocativo, com frases de efeito que instiguem a colaboração do funcionário para o alcance das metas do planejamento, por exemplo: E você? O que você pode fazer para ajudar essas pessoas?

\section{CONCLUSÃO}

O projeto proporcionou conhecer a experiência do colaborador com a comunicação interna e o quanto a sua falha prejudica no entendimento dos principais objetivos estratégicos da organização e consequentemente na dificuldade em alcançar metas, já que as equipes não entendem o planejamento estratégico e seus produtos.

A abordagem Design Thinking foi uma ferramenta altamente apropriada para o projeto, pois possibilitou o desenvolvimento de empatia para com esses colaboradores e, dessa forma, desenvolver uma proposta de melhoria que atinja suas principais dores e ganhos sobre o processo de comunicação e disseminação do planejamento estratégico.

O Design Thinking é uma ferramenta que vem sendo utilizada como um meio de desenvolver inovações nas organizações. No ambiente hospitalar ela foi extremamente importante para uma abordagem empática e colaborativa na resolução de problemas, altamente centrada no colaborador e capaz de estruturar uma proposta de melhoria inovadora através da iteração de profissionais de diversos setores e de práticas criativas.

\section{REFERÊNCIAS}


1. Mendes VLP, Cunha FJAP, Teixeira, F. Redes Sociais de Colaboração: A experiência de uma rede de hospitais. In: Teixeira, F. (Org.). Gestão de redes de cooperação interempresariais: Em busca de novos espaços para o aprendizado e inovação. Salvador: Casa da Qualidade: 2005. p. 77-95.

2. Nassar MRF. Comunicação: Políticas e estratégias para área da saúde. In: Congresso Brasileiro de Ciências da Comunicação, 27, 2004, Porto Alegre. São Paulo: Intercom, 2004.

3. Bittar OJNV. Gestão de processos e certificação para a qualidade. Rev Assoc Med Bras. 2000; 1(46):15-34.

4. Aguiar F, Mendes VLPS. Acreditação hospitalar: A comunicação como ferramenta de viabilização de processos e segurança do paciente. In: Congresso Internacional de Qualidade em Serviços e Sistemas de Saúde, 2011, 1, São Paulo. São Paulo: FGV/EAESP, 2011. p.169-175.

5. ANAHP. Associação Nacional de Hospitais Privados. Comunicação no ambiente hospitalar. https://www.anahp.com.br/noticias/comunicacaono-ambiente-hospitalar/.

6. Brown T. Design thinking. Harvard business review. 2008; 86(6):84.

7. Bonini LA, Sbragia, R. O modelo de design thinking como indutor da inovação nas empresas: Um estudo empírico. Revista de Gestão e Projetos. 2011; 2(1).

8. Cardon EC, Leonard S. Unleashing design: planning and the art of battle command. https://www.armyupress.army.mil/Portals/7/militaryreview/Archives/English/MilitaryReview 20120630MC art008.pdf

9. Giannini L. Comunicação e Relacionamento: A criação de personas para atuação de instituições públicas em redes sociais digitais. Revista Temática. 2016; 12(11).

10. Liedtka J; Ogilvie T. Designing for Growth: A Design Thinking Toolkit for Managers. Columbia University Press, 2011.

Recebido: 09 de fevereiro de 2019. Aceito: 02 de junho de 2020

Correspondência: Caroline Maiolini Mizael. E-mail: carolmmizael@gmail.com

Conflito de Interesses: os autores declararam não haver conflito de interesses. 
(C) This is an Open Access article distributed under the terms of the Creative Commons Attribution License, which permits unrestricted use, distribution, and reproduction in any medium, provided the original work is properly cited 